\title{
Magnetic properties and magnetic structure of the quasi one-dimensional antiferromagnet $\mathrm{Cu}_{2}\left(\mathrm{MoO}_{4}\right)\left(\mathrm{SeO}_{3}\right)$
}

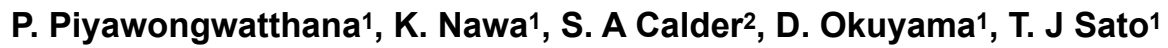 \\ ${ }^{1}$ Institute of Multidisciplinary Research for Advanced Materials, Tohoku University, Sendai 980-8577, Japan, ${ }^{2 N e u t r o n ~ S c a t t e r i n g ~}$ \\ Division, Oak Ridge National Laboratory, Tennessee 37831, USA \\ piyawongwatthana.pharit.p3@dc.tohoku.ac.jp
}

One-dimensional spin chain systems have been attracting renewed interest in terms of a magnon/spinon-band splitting, which is found in $\mathrm{Cu}_{2} \mathrm{~V}_{2} \mathrm{O}_{7}[1]$ and $\mathrm{Cs}_{2} \mathrm{CuCl}_{4}$ [2]. Nonreciprocal propagation of such quasiparticles is of growing interest since it may be used for spintronics device realization. A key parameter for the band splitting is an intrachain antisymmetric Dzyaloshinskii-Moriya interaction. To expand the variety of materials that can exhibit the band splitting, we have further searched for a possible quasi one-dimensional antiferromagnet. $\mathrm{Cu}_{2}\left(\mathrm{MoO}_{4}\right)\left(\mathrm{SeO}_{3}\right)$ is a candidate quasi one-dimensional compound, which crystallizes in a monoclinic system (space group $P 2_{1} / c$ ) with the unit cell parameters $=104.675(12)^{\circ}, a=8.148(5) \AA, b=9.023(5) \AA$, and $c=8.392(5) \AA$ [3]. The $\mathrm{Cu}^{2+}$ ions are connected via edges of $\mathrm{CuO}_{5}$, forming armchair-like chains along $c$-axis with three different bond lengths, $3.186 \AA, 2.973 \AA$, and $3.149 \AA$. Due to the lack of local inversion symmetry between the shortest $\mathrm{Cu}(1)-\mathrm{O}-\mathrm{Cu}(2)$ bonds, the active $\mathrm{DM}$ interactions between these bonds could be expected.

We have performed single crystal magnetic susceptibility measurements and a neutron powder diffraction to characterize this compound. Figure 1 shows the magnetic susceptibility measured under the applied field $H=10 \mathrm{kOe}$ along the $a-, b$-, and $c$-axes. The magnetic susceptibility shows an anomaly at $T_{\mathrm{N}} \sim 23 \mathrm{~K}$ in all directions. The temperature dependence of the magnetic susceptibility shows a broad maximum near $50 \mathrm{~K}$ indicates a low-dimensionality and short-range correlation. The magnetic susceptibility was fit between $130 \mathrm{~K}<T<$ $300 \mathrm{~K}$ to the Curie-Weiss law and the Curie-Weiss temperature $\Theta=-68(1) \mathrm{K}$ was obtained. The negative Curie-Weiss temperature confirms that the dominant exchange interaction between $\mathrm{Cu}^{2+}$ ions in $\mathrm{Cu}_{2}\left(\mathrm{MoO}_{4}\right)\left(\mathrm{SeO}_{3}\right)$ is antiferromagnetic. Moreover, the magnetic susceptibility strongly depends on the crystallographic direction suggesting that the $g$-factor in this compound is anisotropic. A sharp drop in the susceptibility only along $c$-axis is observed suggesting that the majority spins align along this direction.

A neutron powder diffraction experiment was carried out on HB2A powder diffractometer at High Flux Isotope Reactor (HFIR), Oak Ridge National Laboratory (ORNL). Ge(113) monochromator was used to select neutrons with $\lambda=2.41 \AA$. Magnetic Bragg reflections were observed below $T_{\mathrm{N}}$ on top of the structural reflections indicating the magnetic propagation vector $q=\left(\begin{array}{ll}0 & 0\end{array}\right)$. Magnetic structure analysis was performed using the representation analysis method; we found that $\mathrm{Cu}_{2}\left(\mathrm{MoO}_{4}\right)\left(\mathrm{SeO}_{3}\right)$ orders with antiferromagnetic structure, where the magnetic moments are mostly parallel (or antiparallel) to the chain direction. The resulting magnetic structure is shown in figure 2.

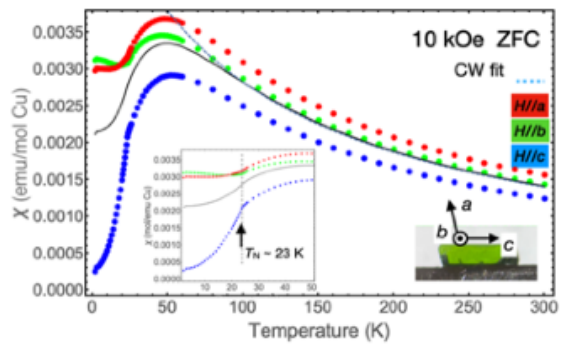

Figure 1. Magnetic susceptibility measured in the single crystal of $\mathrm{Cu}_{2}\left(\mathrm{MoO}_{4}\right)\left(\mathrm{SeO}_{3}\right)$ with applied field of $10 \mathrm{kOe}$ along the three crystallographic directions. Black line is the average magnetic susceptibility.

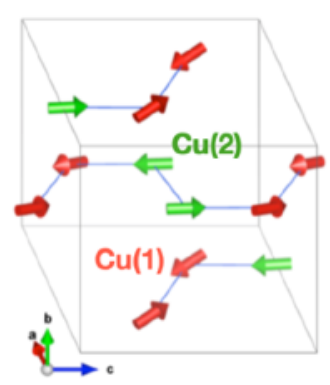

Figure 2. The magnetic structure of $\mathrm{Cu}_{2}\left(\mathrm{MoO}_{4}\right)\left(\mathrm{SeO}_{3}\right)$ deduced from powder neutron diffraction and magnetic susceptibility measurements. The arrows represent the magnetic moment of $\mathrm{Cu}^{2+}$ ions.

[1] G. Gitgeatpong, et al., Phys. Rev. Lett. 119, 047201 (2017)

[2] K. Yu. Povarov et al., Phys. Rev. Lett. 107, 037204 (2011)

[3] S. Y. Zhang, H. et al, Inorg. Chem., 48 (24), 11809-11820 (2009)

Keywords: Magnetic structure, one-dimensional spin system

Acta Cryst. (2021), A77, C962 